\title{
COMPUTATIONAL MODELING OF BUBBLE GROWTH FROM A SUBMERGED ORIFICE WITH CONSTANT AIRFLOW RATE
}

\section{SACHIN KUMAR* \& RAJ KUMAR SINGH}

Department of Mechanical Engineering, Delhi TechnologicalUniversity, Delhi (India)

\begin{abstract}
This study investigated the single bubble evolution in immersed orifice with a view the effect of bubble shape changes with time and variation of pressure in the liquid during the bubble move upward during the bubble growth, pinch-off region and detachment has been investigated by using computational fluid dynamics. The orifice diameter varies from $0.5 \mathrm{~mm}$ to $2 \mathrm{~mm}$. Measurement of an arc is created and Young-Laplace equation has used to the estimation of the pressure of liquid varies in the gas liquid surface where the bubble is evolved. The present wok is focused on different wetting conditions at low gas flow rate and wetting region of the orifice with orifice base was studied for the working fluid of air and water at $20^{\circ}$. Analytical simulations of bubble formation with constant flow rate and constant gravity where the formation of bubble occurred when the inertia force is going to be leading force which was coupled with level set and volume of fluid (CLSVOF). Simulation data on the transition of bubble formation by using the capillary number with different wetting condition.
\end{abstract}

KEYWORDS: Computational Fluid Dynamics, Multiphase, Bubble Formation

Received: Oct 23, 2020; Accepted: Nov 13, 2020; Published: Dec 10, 2020; Paper Id.: IJMPERDDEC202023

\section{INTRODUCTION}

The bubble evolves from the immersed orifice has a most useful factor in a different industries usage like chemical and bio-chemical reaction and in the various other equipment worldwide used in metal casting or metal production industry and big industries of casting used to suppress the bubble formation at casting process and spend crore of rupees to diminish the bubble formation during casting process. Bubble evolution process is a state transformation process like during water boil and other metallurgical process. Due to its industrial application and day-to-day life application the bubble evolution is a great research topic of researchers that have been widely used in the last 5 decades. Different analytical models Kumar and Kuloor, 1970 and Kulkarni and Joshi, 2005) studied the two model which is one-stage model and two-stage model was developed and two- stage model give more precise result as compare to one-stage model, to give the volume of a bubble formed by solving the different forces developed when the bubble move forward like buoyant force, pressure force and gas momentum and constraining forces acting on a bubble e.g. surface tension ,drag and inertia forces. The analytical model predicts the bubble volume in this state (Gaddis and Vogelpohl,1986; Jamiamlahmadi et.al., 2001. Surface tension is near to zero except at the initial stage of evolution of bubble and inertia force get to be exceed from the surface tension force. Earlier models of Davidson and Schuler (1960a, b) bubble evolution in viscous and non-viscous liquids, better models suggested by use of two-stage and non-spherical models (Tsuge,1986). The present work main center of study on the bubble evolution process but also provide all data about the conditions under which the formation state changes.is used based on the concept of a fractional. The present work considers wettability of the orifice plate. Buwa et al. (2007) studied the bubble formation on an orifice of $2-10 \mathrm{~mm}$, combined with the numerical 
simulation. Gerlach et al. (2007) found the transformation from initial bubble to second bubble formation in their numerical simulation. (Quan and Hua, 2008; Das and Das,2009; Chakraborty et al., 2009; Ohta et al., 2011; Chakraborty et al., 2011) on nozzle and orifice results are motivational. Nowadays, software work is preferred because it is easy to get the result in a better way so recent years many analytical results of bubble formation and variation of in liquid movement have been organized by volume of fluid (VOF) used to deal with intricate geometry. Zhou et al. (2011) take VOF method to analyze the bubble evolution, rate of increase of volume and disengagement of bubble with the nozzle. This study main focused on the outcome of wet region of the wall where the bubble gets evolved. Researcher put the force to make this study for clear understanding of bubble formation and how to bubble shape varied with time and how the velocity variation occurs when the liquid gets disturb.

\section{PROBLEM DESCRIPTION}

The evolution and disengagement of bubble at immersed orifice under water and gas flow through the passage of nozzle and cubic measure of bubble increases continuously. When the buoyant force exceeds the drag force the bubble detaches and rises upwards. The process of evolution and disengagement is taking to be symmetric where by center of the bubble at the $(\mathrm{x}, \mathrm{y})$ coordinate system. assumed to be axisymmetric, we take the constant $\mathrm{R}_{0}$ radius of bubble at nozzle and we do not put any pressure in our simulation so we say that it is not depend on pressure variation on the bubble. This is known as the constant flow state and it contradict the constant pressure state (Clift et al., 1978). The various properties of the liquid state are the density $\rho_{l}$ and the viscosity $\mu_{l}$, the corresponding properties of the gas state being $\rho_{g}$ and $\mu_{g}$. The surface tension $\sigma$ value taken to be constant $0.072 \mathrm{~N} / \mathrm{m}$. Gas-liquid and solid phase occur in the three-phase contact line and we applied a static contact angle movement to describe the movement of bubble with respect to triple contact line

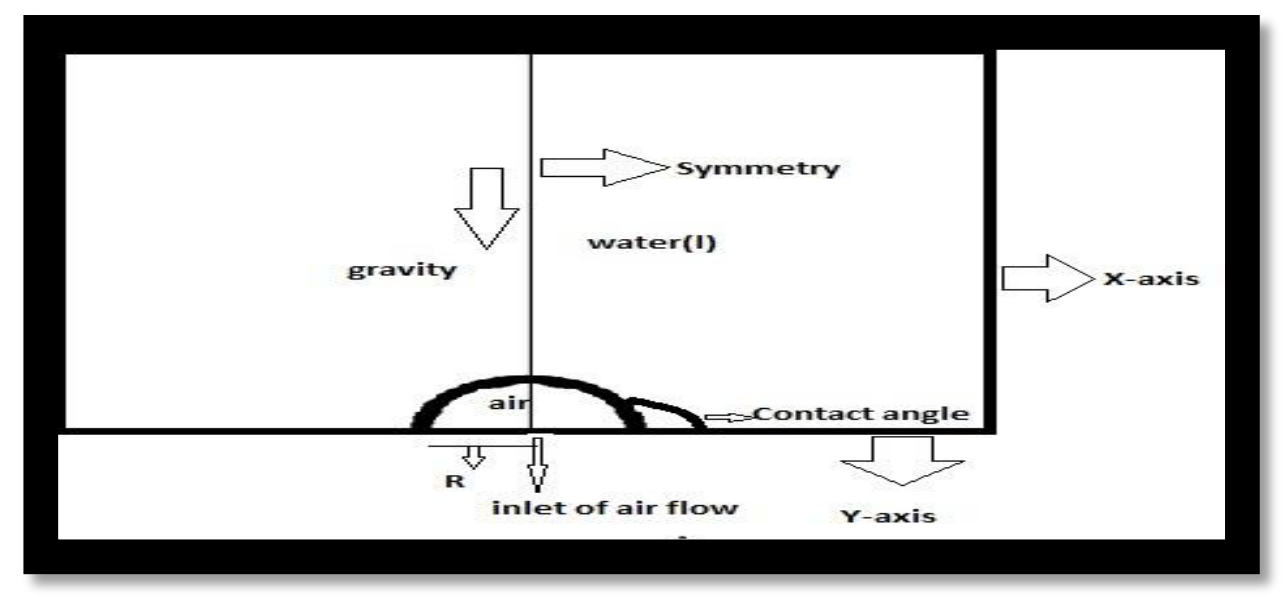

Figure 1: 2-d Domain for Bubble flow Water-air Interface

upward and it is called a gas-liquid periphery. Initially, the bubble phase is fixed in the nozzle that the contact line of the nozzle rim. We make many assumptions in the bubble flow so that we calculate the number of forces acting on the bubble. Since we know the gas flow rate.An analytical solution of this study, the continuity and Navier-Stokes equations for an incompressible Newtonian fluid are clarified with the defined parameters. We demonstrate the bubble area in different phase initially the bubble at the triple contact line than after it becomeswetwhen it move and gas density are very negligible as compare to act forces. We assumed to be reduced the gas flow rate on the bubble and the equilibrium between the force.

$$
\sum F-F_{B}+F_{C P}+F_{C}+F_{D}=0
$$


For the analytical solution of the problem, Navier-Stokes and continuity equations are used to calculate the incompressible Newtonian fluidunder certain conditions. When the bubble moves forward than certain forces will act on the bubble periphery and dimensionless number are calculated with the help of the forces which act on the bubble. Initially, the bubble is evolved than the equilibrium will be established between the buoyant force and surface tension i.e.,

$$
2 \pi R_{0} \sigma=V_{B}\left(\rho_{l}-\rho_{g}\right) \mathrm{g}
$$

Where $\mathrm{g}$ is the gravitational force act opposite to the bubble movement. The fraction of two forces is defined by the Bond number

$$
\mathrm{Bo}=\frac{\rho_{l} g R_{0}^{2}}{\sigma}
$$

Where $\rho_{g}$ was negligible, since $\rho_{g}<<\rho_{l}$ here, Eq. (1) indicates that at quasi-static flow rate volume rate of bubble expanded with the nozzle semidiameter and also surface tension but it decreases with the increase of density change. The viscous force developing on the bubble nearly equal to the drag force when the bubble gets to be expanded. Here, in the domain the bubble size so the surrounding water velocity gets change and this velocity can be calculated by the $\dot{Q} / V_{B}^{2 / 3}$ ,appearing in a viscous force of the order $Q \mu_{l} / V_{B}^{1 / 3}$ where $\dot{Q}$ is the rate of flow of air. The division of viscous to capillary forces is predicted by the capillary number.

$$
\mathrm{Ca}=\frac{\mu_{l} \dot{Q}}{\sigma R_{0}^{2}}
$$

If we compare the viscous force with the capillary force which is proportional to the gas flow rate and viscosity. The fraction of capillary number and the bond number is $\mathrm{Ca} / \mathrm{Bo}^{1 / 3}$.The capillary and Bond numbers were taken in the present work are $C a, B o$ and Reynolds number $\operatorname{Re}=\rho_{l} \dot{Q} /\left(\mu_{l} R_{0}\right)$.The term $1 / B o^{1 / 3}$ used in the present work is 1.45 where by values of $\mathrm{Ca}$ are below 0.1, e.g. for the standard working fluids ( air and water) under the conditions $R_{0}=1 \mathrm{~mm}$ and $\dot{Q}=$ $100 \mathrm{ml} / \mathrm{min}, \mathrm{Ca}=0.023$. Hence the buoyancy and capillary force are involved in all the cases where the flow rate of gas is considered. This was giving a clear understanding of the static contact angle $\varphi_{s}$ at the three-phase contact line at the base of the domain where orifice/nozzle is fixed triple-phase touch at the orifice plate. As we saw in the analytical results show that the wetting region of the passage(orifice) base may have a great impact on the bubble dimensions with the flow rate of gas. Forces developed when the bubble is moving upwards.

\section{ANALYTICAL MODELING}

In this study, the mass conservation laws and momentum of incompressible Newtonian liquid are as follow:

$$
\begin{aligned}
& \nabla \cdot u=0 \\
& \frac{\partial \rho(H) \vec{u}}{\partial t}+\nabla \cdot\left(\rho(H) \overrightarrow{(u \vec{u})}=-\nabla p+\rho(H) g+\nabla\left(\mu(H)\left(\nabla \vec{u}+(\nabla u)^{T}\right)\right)+F_{S}+\rho \vec{g}\right.
\end{aligned}
$$

Where surface tension force term $F_{S}, \mathrm{p}$ is the pressure, $\mathrm{g}=(-\mathrm{g}, 0)$ is the gravity force whichacts in opposite direction with the bubble direction, $\rho(H)$ and $\mu(H)$ are the density and dynamic viscosity, defined by

$$
\begin{aligned}
& \rho(F)=\rho_{l} \mathrm{H}+\rho_{g}(1-\mathrm{H}), \\
& \mu(F)=\mu_{l} \mathrm{H}+\mu_{g}(1-\mathrm{H}) .
\end{aligned}
$$


The subscript $(l)$ and $(g)$ give the liquid and gas phase, respectively

$$
F_{s}=\sigma k(\varphi) \nabla H(\varphi)
$$

Surface tension $(\sigma) \mathrm{k}(\varphi)$ is the simple average arc formed mean by the interface, $\varphi$ (level-set function) and, Heavy side function $\mathrm{H}(\varphi)$.

$$
\mathrm{H}(\varphi)= \begin{cases}1 & \text { if } \varphi>0 \\ 0, & \text { otherwise }\end{cases}
$$

The level-set function $\varphi$ is defined with $\mathrm{F}$, because the number of formulae arise for this calculation and simple average $\operatorname{arc} k(\varphi)$ in the surface tension force term, Eq. (9). $\varphi$ is described as the function of distance which is measured from the periphery (see, e.g., Sussman et al., 1994).Theliquid region is that where $\varphi>0$ that region is the liquid region and where $\varphi<0$ that region is the gas region. The periphery is constantly given by the $\varphi=0$ contours. The average arc in Eq (8)

$$
k=\nabla \cdot \frac{\nabla \varphi}{|\nabla \varphi|}
$$

The surface tension force is used to calculate the volume of the bubble by using interface curvature $k$ and is used in a fixed region around the periphery by using the $\delta$ function.

$$
\begin{aligned}
& F_{\sigma}=\sigma k(\varphi) \delta(\varphi) \nabla \varphi \\
& \delta(\varphi)= \begin{cases}0 & , \text { if }|\varphi|>0 \\
\frac{1}{2}\left(1+\cos \left(\frac{\pi \varphi}{\epsilon}\right)\right), & \text { if }|\varphi| \leq \epsilon\end{cases}
\end{aligned}
$$

The quick scheme (Leonard,1970) is available for the advection equation in the LS method, which is third-order scheme while the calculation of different equations again and again by using the WENO scheme (Liu et al., 1994) with 25 artificial iterations. The simple law (Patankar and Spalding, 1972) is used to pressure-velocity coupling in the continuity and momentum equations.

\subsection{Coupling VOF with LS (CLSVOF)}

Coupling VOF with LS used to conserve the interface profile with volume fraction $\alpha$, and LS function has no conservation of mass because LS function lost distance parameter after solving advection equation. Both methods have equally benefit and counter benefit so we coupled both methods.

\subsection{Problem Definition}

\section{Computational Setup}

The bubble evolution from a nozzle of $1 \mathrm{~mm}$ radius in a container of filled with water and the airflow rate is $100 \mathrm{ml} / \mathrm{min}$ applied in the nozzle where the bubble is formed with constant flow rate $\dot{Q}$. At the inlet, a laminar velocity profile $\mathrm{x}$ direction is the symmetric region as sketched in Fig. where $R_{C}$ and $D_{e q}$ are the nozzle/orifice radius and equivalent diameter of a bubble, respectively. The boundary condition at the mid of line $\mathrm{x}=0$ and at the left and right extreme position $\mathrm{r}=\mathrm{R}$ as symmetric extreme conditions i.e., $\mathrm{u}=0$ and,Where $V_{0}=\dot{Q} / \pi R_{0}^{2}$ is the mean velocity at the nozzle/orifice.

Thedimensionless number is based upon the flow properties of the gas. We should take the interface of the threephase contact line at the orifice plate. In this study, we take different contact angle with wetting conditions and non-wetting 
conditions, this means the contact angle $\varphi<\varphi_{s}$, the bubble bottom side starts to increase externally and the moving contact lines keeps the constant contact angle $\varphi=\varphi_{s}$, which does not depend on the bubble movement and the direction of the bubble. We fixed the contact line for $\varphi>\varphi_{s}$ and using the fixed contact angle during the contact line movement by fixing all the domain confined condition $\partial \varphi / \partial z=-\cos \varphi_{s}$. The computational domain describes the fig. as $\mathrm{R}=5 D_{e} / 2$ and $\mathrm{Y}=10 D_{e}$, which is nearly equal to the area $10 \times 20 \mathrm{~mm}^{2}$ where $D_{e}$ is equivalent to spherical bubble diameter. The cell size of the mesh is $0.25 \mathrm{~mm}$ this step size is taken by (Buwa et al., 2007 ; Chakraborty et al., 2009 ; Gerlach et al., 2007). The time step taken in this study was $1 \times 10^{-6}$ to satisfy the CFL constraints provided.

\section{RESULTS}

\subsection{Influence of Wetting Conditions}

We are here study the constant flow of air through the orifice/nozzle $\dot{Q}$, the orifice radius $R_{0}$ and we provide a different static contact angle $\varphi_{s}$ (wet region) on the bubble formation is predicted by the help of static contact angle. In these studies, we should keep the properties of fluid were kept constant at the standard value of an air-water system at $20^{\circ} \mathrm{C}$. We are restricted in numerical efforts orifice radii at $R_{0}=1$ and $1.5 \mathrm{~mm}$ were studied. We are studied in this investigation $100 \mathrm{ml} / \mathrm{min}$ was gas flow rate where $\varphi_{s}=50^{\circ}, 0^{\circ}$ (wettingliquid) and
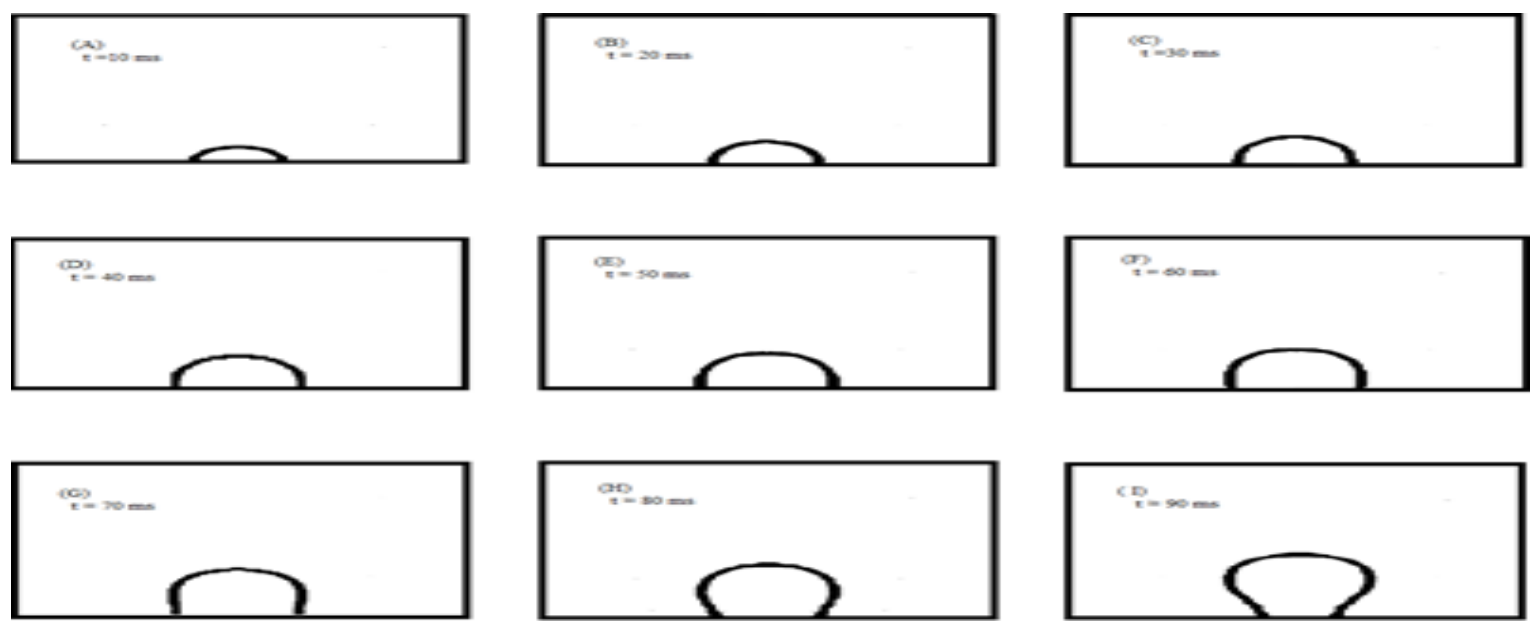

Figure 4.1.: Different Instant of Bubble Formation, $\varphi_{s}=0^{0}, R_{0}=1 \mathrm{~mm}$ and $\dot{Q}=100 \mathrm{ml} / \mathrm{min}$.
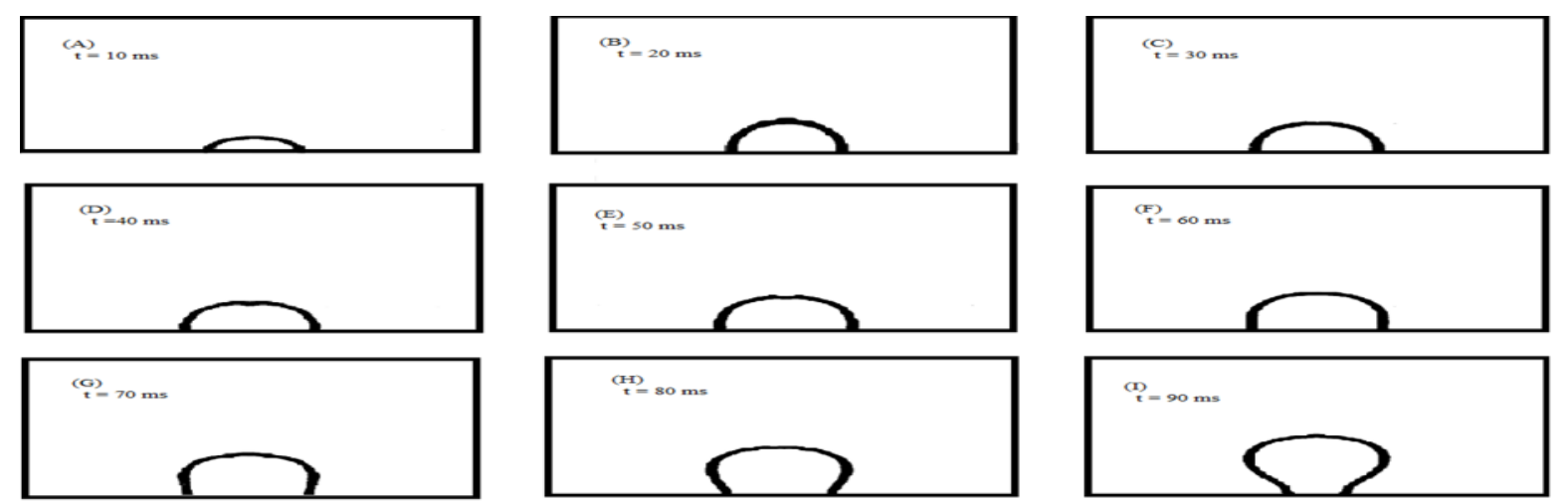

Figure 4.2: Different Instant of Bubble Formation at $\varphi_{s}=50^{\circ}, R_{0}=1 \mathrm{~mm}$ and $Q^{\circ}=100 \mathrm{ml} / \mathrm{min}$. 

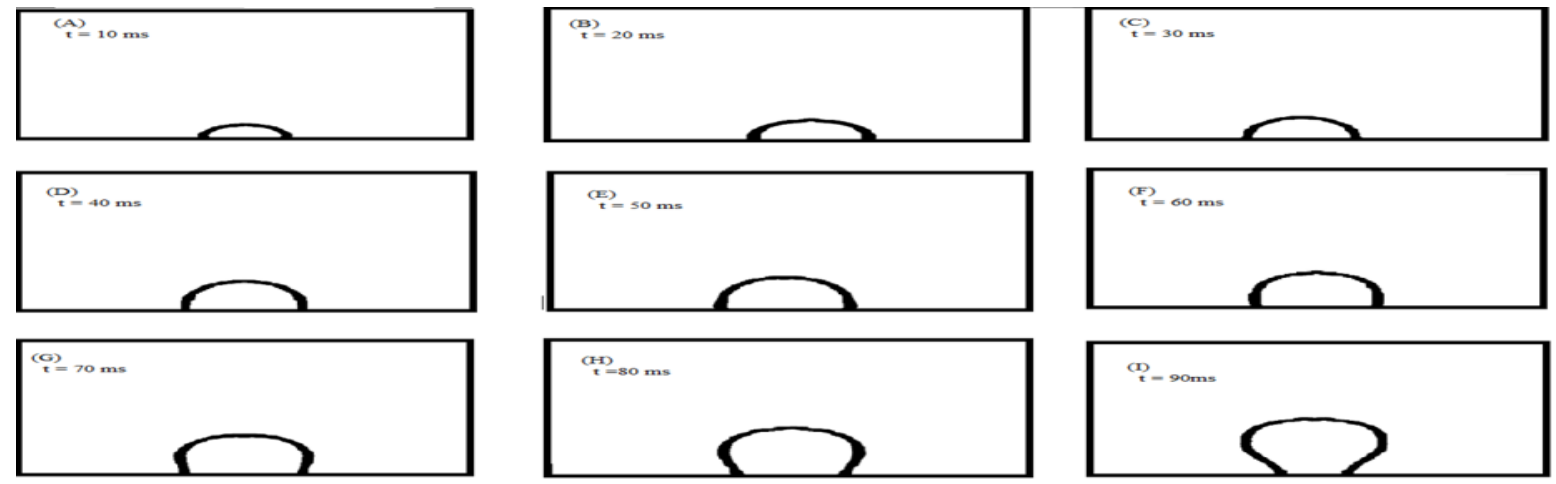

Figure 4.3: Different Instant of Bubble Formation $\varphi_{s}=110^{\circ}, R_{0}=1 \mathrm{~mm}$ and $Q=100 \mathrm{ml} / \mathrm{min}$

\subsection{Contact Angle Variation with Time}

The contact angle variation is change with each time step initially the bubble contact angle is high and when the initially $\mathrm{t}=0$ and when the time steps is increasing with time than contact angle with time decreases and when the contact angle is initially decreases where the necking region begins to start where contact angle decreases to start and after that the contact angle begins to increase their variation of contact angle graph like parabola .

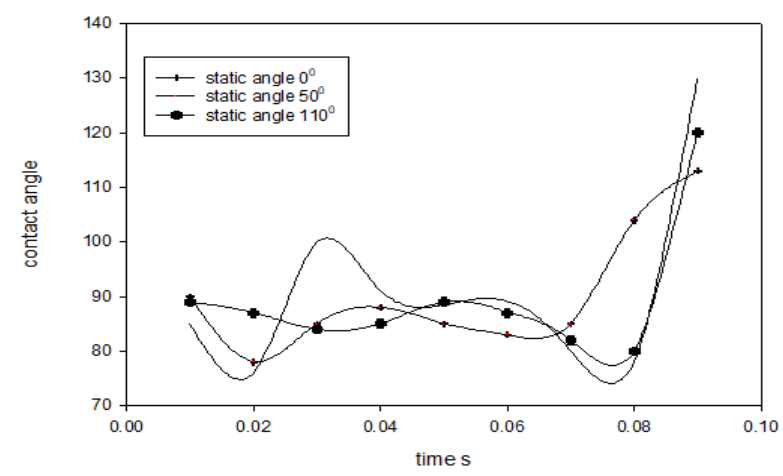

Figure 4.4: Contact Angle Variation with Time

The bubble growsupwards as time-lapse. We take the different static contact angle $\varphi_{s}=0^{0}, 50^{\circ}$ and $110^{\circ}$ and check the variation of angle with the orifice rim of the plate when the bubble grows upwards, Initially contact angle decreases with time and when the bubble necking region begins than the contact angle of the bubble with orifice rim increases drasticall

\subsection{Bubble volume Variation with Time}

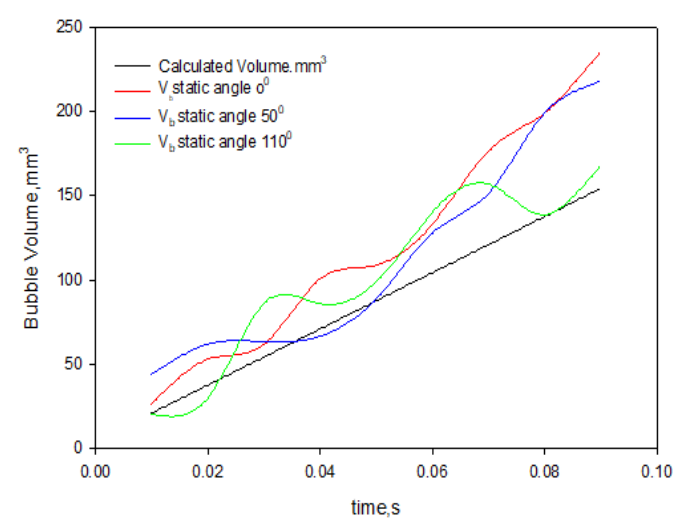

Figure 4.5: Bubble Volume with Time 
The bubble dimension in $\mathrm{mm}^{3}$ variation concerning time, we make the assumption as a bubble volume take as a spherical volume which we take as linear variation relation i.e., $\mathrm{V}(\mathrm{t})=V_{0}+Q_{g} t$ this relation we consider to calculate the bubble volume in each time step. We take as a reference paper to take this assumption (bubble generation in quiescent and co-flowing fluid) and we consider the bubble volume consider as spherical shape. This linear line is generated from the above linear relation and the other volume is calculated volume at each time step at different static contact angle $\varphi_{s}$. Volume (1) is predicted for $\varphi_{s}=0^{0}$ calculated volume for simulation data, volume (2) is predicted for the $\varphi_{s}=50^{0}$ calculated volume for generated data by simulation and volume (3) is predicted for the $\varphi_{s}=110^{0}$ calculated volume for generated data for the simulation.

\subsection{Bubble Diameter Variation with Time}

Diameter variation concerning time as the bubble volume varies with time than bubble diameter is change with respect to time than we calculate the bubble diameter variation concerning time we take the relation to calculate the bubblediameter variation with time $D_{b}(t)=D_{0}^{3 / 2}+\left(6 Q_{g} / \pi\right) t^{1 / 3}$

Hence, we calculate the bubble diameter variation with respect to time using above relation take from reference paper (study of bubble formation under constant flow condition

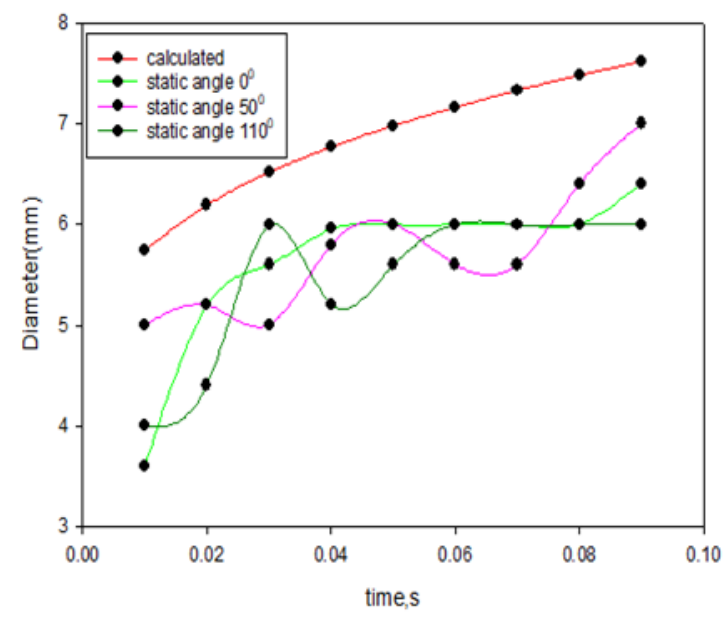

Figure 4.6: Change in Diameter with Respect to Time

\begin{tabular}{|c|c|c|c|c|}
\hline$\varphi_{S}^{0}$ & $\begin{array}{c}V_{b}\left(\mathrm{~mm}^{3}\right) \\
\operatorname{VOF}(100 \mathrm{ml} / \mathrm{min})\end{array}$ & $\begin{array}{c}V_{b}\left(\mathrm{~mm}^{3}\right. \\
\operatorname{VOF}(10 \mathrm{ml} / \mathrm{min})\end{array}$ & $\begin{array}{l}\text { Analytical data } \\
\text { (Gerlach et al. } \\
(2005) Q \stackrel{\circ}{\rightarrow} 0\end{array}$ & $\begin{array}{l}\text { Analytical data } \\
\text { Fritz }(1935) Q \stackrel{\leftrightarrow}{\rightarrow} 0\end{array}$ \\
\hline 0 & 190.4 & 39 & 35 & 0 \\
\hline 50 & 217.9 & 39 & 35 & 12 \\
\hline 110 & 167.82 & 139 & 126 & 126 \\
\hline
\end{tabular}

We made table to compare different result for different static contact angles $\varphi_{s}$ at an orifice of $R_{0}=1 \mathrm{~mm}$ under gas flow rate $100 \mathrm{ml} / \mathrm{min}$

As we saw in the simulation the bubble volume increases with increases in each timestep we plot the graph volume v/s time and we calculate the volume in each time step, initially the surface tension $F_{c}$ developing on the downward direction and surface tension force acting on the periphery of the bubble after some time steps bubble volume increases due to which buoyancy force $F_{b}$ also increases as a comparison to capillary forces due to which bubble moves upward and 
bubble periphery widening due to surface tension of bubble and as the bubble moves upwards the necking region is going to start formed and we the snapshot the necking region is going to begins almost $0.07 \mathrm{sec}$ near to start in each bubble static contact angle but the diameter in each contact angle is different due to surface tension varies concerning contact angle because surface tension force is usually expressed in the form of $2 \pi R_{0} \cos \theta$. As we calculate the different surface tension in each different time step with different contact angles.

\section{Pinch-off time}

Pinch-off time in each static contact angle $\varphi_{s}=0^{0}, 50^{\circ}$ and $110^{\circ}$ near to $0.09 \mathrm{sec}$ but at that timestep that the base diameter is vary at each static contact angle $\varphi_{s}=0^{0}$ the base diameter is $1.6 \mathrm{~mm}, \varphi_{s}=50^{0}$, the base diameter is $1 . .4 \mathrm{~mm}$ and $\varphi_{s}=$ $110^{\circ}$ is $1.5 \mathrm{~mm}$ and we see in this simulation the base diameteralso varies alongside the variation of the static contact angle $\varphi_{s}$ and due to which capillary force inertia force and surface tension force is varied as the bubble dynamics

\section{Bubble volume with contact angle}

Bubble volume varies with the contact angle and we calculate the bubble volume in different static angle $\varphi_{s}=0^{0}$, the bubble dimension $\left(\mathrm{mm}^{3}\right)$ at the time step 0.09 is $235.4 \mathrm{~mm}^{3}, \varphi_{s}=50^{0}$, the bubble volume at the time step $0.0 .9 \mathrm{~s}$ is $217.9 \mathrm{~mm}^{3}$ and $\varphi_{s}=110^{0}$ the bubble dimension $\left(\mathrm{mm}^{3}\right)$ the time step $0.09 \mathrm{~s}$ is $167.82 \mathrm{~mm}^{3}$ and we get the result that the bubble volume is varied with the static contact angle $\varphi_{s}$ and the bubble volume increase with decrease with static contact angle $\varphi_{s}$. And this is easily seen in the above plot.

\section{CONCLUSIONS}

The result of the numerical study of bubble formation at the single immersed orifice and constant inflow condition $(100 \mathrm{ml} / \mathrm{min})$ was presented. Bubble volume increases with decreasing static contact angle $\varphi_{s}$. The bubble volume increases almost linearly at each static contact angle $\varphi_{s}=0^{0}, \varphi_{s}=50^{\circ}$ and $\varphi_{S}=110^{\circ}$ as we compare the result $10 \mathrm{ml} / \mathrm{min}$ with the $100 \mathrm{ml} / \mathrm{min}$ then I will get that the bubble volume increases with increases good wetting condition but if we move from good to the poor wetting condition than the bubble volume decreases at the pinch-off time that predicts the bubble volume at $100 \mathrm{ml} / \mathrm{min}$ is increases at good wetting condition but it decreases when it moves from good to poor wetting condition and surface tension play an important role to move the bubble and bubble periphery widening and detachment

\section{ACKNOWLEDGEMENTS}

The authors are highly thankful to the Centre for Hydraulic Machinery and Department of Mechanical Engineering, Delhi Technology University, Delhi (India) for their assistance and facilities in compiling this review manuscript.

\section{REFERENCES}

1. Gerlach, D., Alleborn, N., Buwa, V., \& Durst, F. (2007). Numerical simulation of periodic bubble formation at a submerged orifice with constant gas flow rate. Chemical Engineering Science, 62(7), 2109-2125. doi:10.1016/j.ces.2006.12.061

2. Xie, J., Zhu, X., Liao, Q., Wang, H., \& Ding, Y.-D. (2012). Dynamics of bubble formation and detachment from an immersed micro-orifice on a plate. International Journal of Heat and Mass Transfer, 55(11-12), 3205-3213. doi:10.1016/j.ijheatmasstransfer.2012.02.053

3. Gerlach, D., Alleborn, N., Buwa, V., \& Durst, F. (2007). Numerical simulation of periodic bubble formation at a submerged orifice with constant gas flow rate. Chemical Engineering Science, 62(7), 2109-2125. doi:10.1016/j.ces.2006.12.061 
4. Wen, J., Sun, Q., Sun, Z., \& Gu, H. (2018). The effect of multi-orifice plate configuration on bubble detachment volume. Chinese Journal of Chemical Engineering. doi:10.1016/j.cjche.2018.09.024

5. Muilwijk, C., \& Van den Akker, H. E. A. (2019). Experimental investigation on the bubble formation from needles with and without liquid co-flow. Chemical Engineering Science. doi:10.1016/j.ces.2019.03.026

6. Simmons, J. A., Sprittles, J. E., \& Shikhmurzaev, Y. D. (2015). The formation of a bubble from a submerged orifice. European Journal of Mechanics - B/Fluids, 53, 24-36. doi:10.1016/j.euromechflu.2015.01.003

7. Das, A. K., \& Das, P. K. (2015). Numerical Study of Bubble Formation from Submerged Orifice under Reduced Gravity Condition. Procedia IUTAM, 18, 8-17. doi:10.1016/j.piutam.2015.11.002

8. Zhang, J., Yu, Y., Qu, C., \& Zhang, Y. (2017). Experimental study and numerical simulation of periodic bubble formation at submerged micron-sized nozzles with constant gas flow rate. Chemical Engineering Science, 168, 1-10. doi:10.1016/j.ces.2017.04.012

9. Das, A. K., Das, P. K., \& Saha, P. (2011). Formation of bubbles at submerged orifices - Experimental investigation and theoretical prediction. Experimental Thermal and Fluid Science, 35(4), 618-627. doi:10.1016/j.expthermflusci.2010.11.015

10. Chakraborty, I., Biswas, G., \& Ghoshdastidar, P. S. (2011). Bubble generation in quiescent and co-flowing liquids. International Journal of Heat and Mass Transfer, 54(21-22), 4673-4688. doi:10.1016/j.ijheatmasstransfer.2011.06.010

11. LIU, C., LIANG, B., TANG, S., ZHANG, H., \& MIN, E. (2010). A Theoretical Model for the Size Prediction of Single Bubbles Formed under Liquid Cross-flow. Chinese Journal of Chemical Engineering, 18(5), 770-776. doi :10.1016/s10049541(09)60128-2

12. Loubière, K., Castaignède, V., Hébrard, G., \& Roustan, M. (2004). Bubble formation at a flexible orifice with liquid crossflow. Chemical Engineering and Processing: Process Intensification, 43(6), 717-725. doi:10.1016/s0255-2701(03)00017-5

13. Nahra, H. K., \& Kamotani, Y. (2003). Prediction of bubble diameter at detachment from a wall orifice in liquid cross-flow under reduced and normal gravity conditions. Chemical Engineering Science, 58(1), 55-69. doi:10.1016/s00092509(02)00516-x

14. Walters, J., Davidson, J., 1963. The initial motion of a gas bubble formed in an inviscid liquid. Journal of Fluid Mechanics $17(03), 321-336$

15. Leibson, I., Holcomb, E. G., Cacoso, A. G., \& Jacmic, J. J. (1956). Rate of flow and mechanics of bubble formation from single submerged orifices. I. Rate of flow studies. AIChE Journal, 2(3), 296-300. doi:10.1002/aic.690020305

16. Badam, V. K., Buwa, V., \& Durst, F. (2008). Experimental Investigations of Regimes of Bubble Formation on Submerged Orifices Under Constant Flow Condition. The Canadian Journal of Chemical Engineering, 85(3), 257-267. doi :10.1002/cjce.5450850301

\section{APPENDIX 1.1}

\begin{tabular}{|c|c|c|}
\hline S.No. & Date & Presenters \\
\hline 1 & $13 / 11 / 2020$ & Sachin Kumar \\
\hline
\end{tabular}



